\title{
Aportes de la concepción pedagógica de Francisco Ruiz Sánchez para una teoría motivacional perfectiva ${ }^{1}$
}

\author{
Contributions of the Pedagogical Conception of Francisco Ruiz Sánchez \\ for a Perfective Motivational Theory
}

Recibido: 18 de octubre de 2017 - Revisado: 12 de noviembre de 2018 - Aceptado: 15 de enero de 2019

\author{
María Elisa Di Marco ${ }^{2}$ \\ Analía Inés Portela ${ }^{3}$ \\ Mariela Lourdes González ${ }^{4}$ \\ María Gisella Boarini ${ }^{5}$ \\ Hilda Emilia Difabio de Anglat ${ }^{6}$
}

\section{Resumen}

Este artículo señala algunos elementos para comprender la motivación a partir de la plenitud dinámica, esto es, desde la finalidad misma del acto educativo. La investigación analiza la motivación humana en el proceso de aprendizaje a la luz del pensamiento antropológico de Francisco Ruiz Sánchez. Mediante una hermenéutica de sus escritos publicados e inéditos detallamos los tres aspectos principales del proceso motivacional educativo: (a) el método empleado, (b) la propuesta de objetos buenos, y (c) la concienciación del valor del objeto. Se concluye que la motivación constituye un aspecto relacional en el marco de un proceso en el que interviene la cognición o tendencia intelectual hacia un objeto de conocimiento bueno, presentado de una manera sensible y que logra ser percibido como valioso por el educando.

\section{Palabras clave}

Plenitud dinámica; motivación; objetos perfectivos; metodología; conciencia valorativa.

\begin{abstract}
The present work aimed to indicate some aspects for understanding motivation from the dynamic fullness, that is, from the very purpose of the educational event. This research analyzes human motivation during the learning process in the light of the anthropological thought of Francisco Ruiz Sánchez. Through a hermeneutical methodology of his published and unpublished writings, we detailed the three main elements of the educational, motivational process: a) the method used, b) the proposal of good objects, and c) the awareness of the value of the object. It is concluded that motivation constitutes a relational aspect within the framework of a process in which cognition or intellectual tendency towards an object of good knowledge intervenes. This object has also been presented sensitively and can be perceived as valuable by the learner.
\end{abstract}

\section{Keywords}

Dynamic fullness, motivation, perfective objects, methodology, value consciousness.

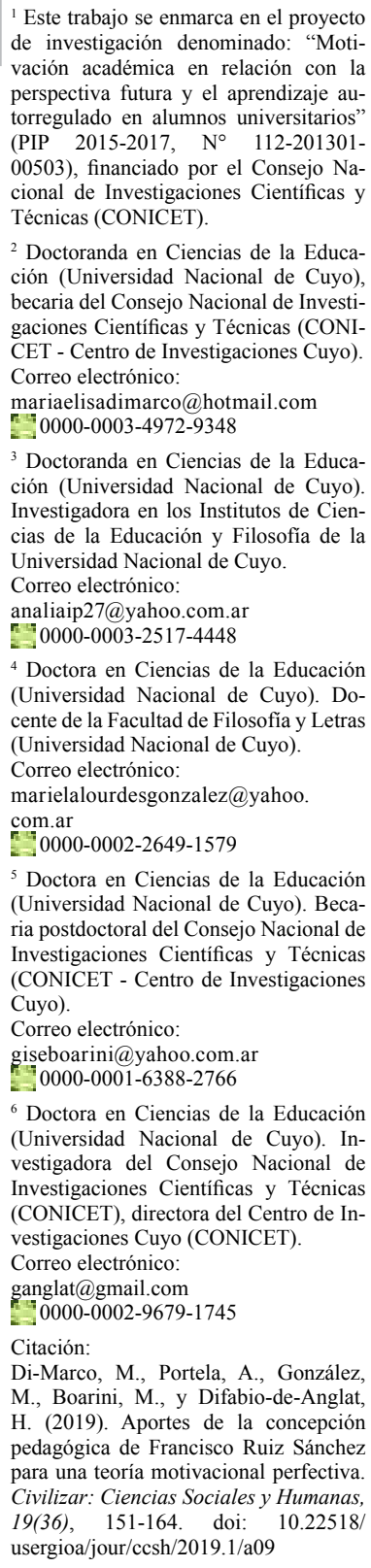

${ }^{1}$ Este trabajo se enmarca en el proyecto de investigación denominado: "Motivación académica en relación con la perspectiva futura y el aprendizaje autorregulado en alumnos universitarios" (PIP 2015-2017, $\mathrm{N}^{\circ}$ 112-20130100503), financiado por el Consejo Nacional de Investigaciones Científicas y Técnicas (CONICET).

${ }^{2}$ Doctoranda en Ciencias de la Educación (Universidad Nacional de Cuyo), becaria del Consejo Nacional de Investigaciones Científicas y Técnicas (CONICET - Centro de Investigaciones Cuyo) Correo electrónico:

mariaelisadimarco@hotmail.com ก7. 0000-0003-4972-9348

${ }^{3}$ Doctoranda en Ciencias de la Educación (Universidad Nacional de Cuyo). Investigadora en los Institutos de Ciencias de la Educación y Filosofía de la Universidad Nacional de Cuyo. Correo electrónico: analiaip27@yahoo.com.ar ก. 0000-0003-2517-4448

${ }^{4}$ Doctora en Ciencias de la Educación (Universidad Nacional de Cuyo). Docente de la Facultad de Filosofía y Letras

(Universidad Nacional de Cuyo).

Correo electrónico:

marielalourdesgonzalez@yahoo.

com.ar

nบ 0000-0002-2649-1579

${ }^{5}$ Doctora en Ciencias de la Educación (Universidad Nacional de Cuyo). Becaria postdoctoral del Consejo Nacional de Investigaciones Científicas y Técnicas (CONICET - Centro de Investigaciones Cuyo).

Correo electrónico:

giseboarini@yahoo.com.ar

giseboarini@y yoon-0001-6388-2766

${ }^{6}$ Doctora en Ciencias de la Educación (Universidad Nacional de Cuyo). Investigadora del Consejo Nacional de Investigaciones Científicas y Técnicas (CONICET), directora del Centro de Investigaciones Cuyo (CONICET).

Correo electrónico: ganglat@gmail.com 19. 0000-0002-9679-1745

Citación:

Di-Marco, M., Portela, A., González, M., Boarini, M., y Difabio-de-Anglat, H. (2019). Aportes de la concepción pedagógica de Francisco Ruiz Sánchez para una teoría motivacional perfectiva. Civilizar: Ciencias Sociales y Humanas, 19(36), 151-164. doi: 10.22518/ usergioa/jour/ccsh/2019.1/a09 


\section{Introducción}

Las teorías de la motivación -motivación de logro (Atkinson, 1964; 1974), de la atribución (Weiner, 2010), del control de la acción (Heckhausen, 1991; Kuhl, 2000; Kuhl y Beckman, 1985), y de la autodeterminación (Deci y Ryan, 1985; 2000; Niemiec, Ryan y Deci, 2010) - abordan el fenómeno de forma psicológico-funcional, sin referencia al desarrollo perfectivo, lo que no niega el valor de las mismas. Empero, más allá de las referidas teorizaciones, el fenómeno motivacional puede ser abordado desde los aspectos perfectivos del ser humano, o sea, a partir de una definición de educación que la asuma como una tarea integral, esto es, considerando al humano como una unidad en la cual participan distintas dimensiones: sensibles, afectivo-tendenciales, intelectuales, moralesvolitivas, etc. Así, la motivación adquiere importancia como uno de los elementos del fin de la educación según la conceptualización en la obra de Francisco Ruiz Sánchez (1962b; 1972; 1973a; 1973b; 1978a; 1978b, entre otras).

Como bien refiere Vázquez (2009):

Las teorías de la motivación más difundidas en las últimas décadas tienen algunos aspectos en común que remiten, a su vez, a una concepción antropológica heredada de la modernidad y en particular del idealismo kantiano. En razón de esta herencia, el momento motivacional se escinde del momento volitivo y la voluntad aparece conceptuada exclusivamente como principio eficiente, dejando de lado el momento previo de carácter pático, de recepción de un bien que suscita una respuesta de amor. (p. 187).

Contra esta dificultad en la que el ser humano aparece escindido, la autora citada presenta una mirada más amplia, dado que a partir de la consideración del círculo del obrar (Vázquez, 2012) o círculo funcional de la vivencia (Lersch, 1966), subraya que el primer paso es el conocimiento, es decir, la aprehensión de una realidad externa al sujeto. Al respecto, expre- sa: "esas realidades son portadoras de cualidades de valor, que si son advertidas por el sujeto, lo afectan, producen una cierta pasión, que la psicología llama respuesta afectiva" (Vázquez, 2012, p. 177). En esta dirección, trasladando el esquema señalado al ámbito pedagógico, el docente es aquel que "muestra" al alumno esa realidad digna de afectarlo, de modo que se produzca la respuesta afectivo-tendencial deseada.

El problema pedagógico-didáctico, entonces, puede abordarse desde diversas aristas: ¿Qué es el valor? ¿Qué condiciones tiene aquello que se concibe como tal? ¿Cómo algo que es valioso en sí mismo puede ser captado por el educando? ¿Cómo hace el docente para mostrar lo que es valioso? ¿Cuáles son las condiciones de esa especie de mostración? etc. Si bien cada uno de estos planteamientos por separado podría suscitar nuevas perspectivas de investigación éticas, psicológicas y gnoseológicas, en el presente trabajo se intenta ahondar en un elemento clave, la mayoría de las veces olvidado, que es la vinculación entre los aspectos motivacionales con el concepto de Plenitud Dinámica (que para Francisco Ruiz Sánchez constituye la finalidad de la educación) y, por tanto, cómo esta puede alcanzarse cuando el educador presenta de manera progresiva y adecuada bienes valiosos para el educando en el orden perfectivo.

En el sentido planteado, se desarrollará la hipótesis de que la propuesta impactante de objetos buenos para el educando por parte del educador, y su valoración consciente positiva de parte del alumno se convierten en aspectos constitutivos del proceso de motivación. En este escrito mostraremos que dicho proceso intervienen varias dimensiones que hacen a la integralidad del sujeto (las afectivo-tendenciales, las morales-volitivas, las intelectuales-espirituales, etc.), las cuales deben orientarse como fin de la educación.

A partir de los aspectos estudiados se concluirá con un análisis sucinto de los elementos que favorecen el proceso de motivación per- 
fectiva del educando, al considerar la finalidad educativa formulada por el profesor mendocino desde una perspectiva que abarca al alumno en sus múltiples dimensiones y tensiones vitales.

En efecto, en la práctica educativa es común advertir que las concepciones de la motivación a las que adhieren, tanto docentes como investigadores, están vinculadas tan solo con el aspecto metodológico-didáctico y que su influencia se constriñe a la apelación sensitiva del sujeto o al mero aspecto volitivo, al abordar su faz estrictamente funcional. Por tanto, en estos planteamientos se observa una subordinación del objeto de conocimiento al método que vacía de contenido ético-pedagógico tanto al proceso de conocimiento como a la tendencia en el proceso motivacional. Así se reduce el aprendizaje a la adquisición de hábitos formales sin sus respectivos objetos, ya sea que se quede en un plano meramente sensible o en el ejercicio de la voluntad como exclusivo principio eficiente.

Por el contrario, se ofrece aquí una perspectiva más comprehensiva, en la que se reconoce la unidad natural del proceso motivacional con un fin educativo, ya que en ambos deben estar integrados, según Ruiz Sánchez, los aspectos sensitivo-afectivos, intelectuales, morales y volitivos del educando. En consonancia, expresa Vázquez (2009):

El núcleo de la motivación es la concepción y adhesión a un fin, en el cual se halla involucrada toda la personalidad en todas sus funciones psíquicas, más aún psico-morales. $\mathrm{La}$ diferencia con el cognitivismo estaría en que la motivación no sería simplemente una idea o razón para obrar sino esa razón aceptada como fin. De aquí se desprenderá el planteo de la educación afectivo - moral como configuración ética de la personalidad, cuyo núcleo es la libre adhesión de la voluntad al bien en cuanto conocido y apreciado como valor - fin. (p. 193).

También en Nuttin $(1973 ; 1980)$ se conceptualiza una naturaleza de la motivación que tiene en cuenta la interacción entre lo cognoscitivo y lo dinámico, lo afectivo y lo tendencial. Desde esta mirada integradora de las potencias y facultades del educando se pretende abordar el presente trabajo en función de lo que Francisco Ruiz Sánchez ha desarrollado como fin de la educación (Di Marco, Portela de Nieto, González, Boarini y Difabio de Anglat, 2018).

Conviene mencionar que la concepción de nuestro autor está sustentada en el supuesto de que la realidad conocida es y existe fuera de nuestro pensamiento y en nuestra inteligencia, de manera intencional, esto es, desde una visión realista. De estas consideraciones se desprenden varios aspectos fundamentales para el ámbito educativo, entre ellos, el reconocimiento de la tensión humana por conocer la verdad en el plano gnoseológico y la tendencia hacia el bien en el plano ético. Ambas tensiones se hallan comprendidas en la teleología de Ruiz Sánchez.

Por otra parte, en el planteamiento del pedagogo argentino, el educando es considerado causa eficiente principal de la educación y el educador causa instrumental y subsidiaria en el proceso educativo, caracterización que se nutre en gran medida en el pensamiento de Aristóteles y Tomás de Aquino.

Dado que el hecho educativo implica distintos aspectos dinámicos, $\mathrm{y}$ la realidad integral del educando supera cualquier análisis parcial, toda teoría educativa debe estar abierta a establecer una estrecha colaboración con otros campos de conocimiento: el ético, el cultural, el social, el político, etc. Por lo mismo, debe tener en cuenta los supuestos teóricos, así como considerar los aspectos existenciales, esto es, circunstanciales, contextuales y subordinantes de la misma práctica educativa en donde se pone en juego in situ el arte prudencial del educador.

Así, este trabajo de índole teórico y hermenéutico, así, de acuerdo con Gadamer (1994; 2015), se estructura en dos partes: esclarecer la finalidad educativa según la entiende F. Ruiz 
Sánchez y detallar los aspectos constitutivos del proceso motivacional, el cual comprende a su vez: (a) la presentación de una metodología adecuada, (b) la propuesta de objetos buenos a la finalidad educativa del sujeto y (c) la concienciación en el educando del valor del objeto en vistas al logro de su plenitud dinámica.

De allí que se concluye que la motivación es un constructo relacional que comprende un objeto de conocimiento bueno o un bien apetecible, intencionalmente presentado como tal y percibido como valioso por el sujeto del acto educativo. El análisis propuesto abre el debate sobre la motivación formalizada en su vínculo con el desarrollo integral perfectivo del educando (es decir, que promueva el desenvolvimiento pleno de sus capacidades y aptitudes acordes a su fin). En este sentido, sin desconocer su relevancia, se dejan de lado otras consideraciones, como su relación con el rendimiento académico o con la teoría de las metas de logros (Elliot y McGregor, 2001) o de la satisfacción de la necesidad de competencia (Cecchini, MéndezGiménez y García Romero, 2019).

\section{La finalidad educativa en Ruiz Sánchez}

Francisco Ruiz Sánchez (1929-1982) fue profesor e investigador en la provincia de Mendoza, Argentina. Su actividad docente se desarrolló de forma intensa a partir de los años 70 en distintas instituciones universitarias del medio regional (Universidad Nacional de Cuyo, Universidad Católica de Cuyo y Universidad Católica Argentina). Allí se desempeñó como profesor de Filosofía de la Educación y de Pedagogía, entre otras disciplinas. Cabe mencionar que fue decano fundador de la sede de la Universidad Católica Argentina en Mendoza.

En sus escritos, el autor ha legado algunas consideraciones importantes sobre los fundamentos de la educación, el fin de la misma, el concepto de educación, la educabilidad, entre otras categorías que pueden fundamentar la reflexión y acción educativas. Su obra aborda tópicos tan viejos como la existencia humana como actuales: ¿Qué es el hombre? ¿Cuál es el fin de la educación? ¿En qué consiste la educación?, entre otros. Fundamentos y fines de la educación, primera edición de 1978, cabe ser mencionado como uno de sus escritos más representativos.

El mérito del autor radica en explicar de manera argumentada y coherente cuáles son los fundamentos y los fines que animan el hecho educativo en sí mismo, más allá del contexto histórico o social y de las particularidades de cada sujeto. De este modo, la profundidad de su obra reúne las condiciones de los filósofos que reflexionan sobre la realidad -en este caso, la educativa- desde sus primeras causas y principios al modo aristotélico.

Si bien el profesor mendocino no realiza propiamente un análisis acerca de la motivación, de su propuesta pedagógica es posible dilucidar los diversos aspectos antropológicos a partir de los cuales nos es posible configurarla. Él sustenta su teoría sobre el fin de la educación en dos principios: "todo agente obra por causa de un fin" y "la potencia se explica -está determinada, especificada- por el acto" (Ruiz Sánchez, 1978a, p. 289). Así pues, la educación va a actualizar al hombre, a perfeccionarlo, desplegando sus potencialidades que lo proyectan a alcanzar su plenitud de ser.

En efecto, en función de lograr la plenitud propia del hombre (Ruiz Sánchez, 1978a, p. 298), el fin de la educación para el autor se halla subordinado al fin del hombre1, "los fines subordinantes, los de la vida humana, determinan y condicionan a los fines de la educación" (Ruiz Sánchez, 1978a, p. 301). En este sentido, tiene en cuenta dos aspectos: uno, la esencia o naturaleza humana, es decir, aquello que al hombre lo hace tal, a partir de lo cual se puede hablar de fines universales de la educación; otro, las particularidades individuales, relacionales, concretas, etc., a partir de las que se puede hablar de fines diferenciados de la educa- 
ción (Ruiz Sánchez, 1978a, pp. 305-306). Sobre tal distinción, señala que el fin de la educación es un estado de plenitud que denomina Plenitud Dinámica, que opone a "la indigencia inicial del hombre y a las carencias en lo humano que potencial, pero naturalmente, puede conservar total o parcialmente toda su vida" (Ruiz Sánchez, 1978a, p. 309).

Esta plenitud como capacidad ordenadora y perfectiva hacia los bienes del hombre confiere sentido a la motivación como estado que favorece el vínculo relacional entre el sujeto de aprendizaje y el objeto bueno o valioso -como lo llamaremos posteriormente-, esto es, un objeto digno de conocimiento o afectación. De aquí se puede inferir una primera conclusión: no todo auxilio que se dice educativo es perfectivo, la condición es que ordene el dinamismo del ser humano para tender a los bienes que lo perfeccionan y, así, lograr ese estado de Plenitud Dinámica. No obstante, para que se produzca, deben darse ciertas condiciones tratadas más adelante.

Digamos primero que Ruiz Sánchez utiliza dos conceptos que ayudan a interpretar el fin de la educación que propone: falibilidad y perfectibilidad.

La falibilidad, que podríamos denominar como el primer eje del pensamiento del autor, implica imperfección. Dado que el hombre nace incompleto e inacabado - con la sola vida no le vienen dadas todas las habilidades y conocimientos necesarios para vivir-y requiere ir haciéndose en el camino de su existencia única, singular y concreta. Pero, en este "hacerse" hay posibilidad de errar, de equivocarse, de fallar y hasta de frustrarse:

¿Quién no tiene en su vida un error, sea en su razonamiento, sea en su conducta? O mejor, ¿quién no tiene la experiencia interior de la posibilidad de equivocarse? ¿Quién no ha visto a otros errar en un razonamiento, en una afirmación, en su conducta? (Ruiz Sánchez, 1978a, p. 145).
La filosofía de la educación del autor está transida de este concepto ${ }^{2}$, pues "esta peculiaridad se halla en la raíz de la necesidad de la educación, de la que es determinante" (1978a, p. 145). Como bien expresa:

Lo extraño es que el hombre sea el único que, estando esencialmente ordenado en su intimidad, puede estar efectivamente desordenado; el único que, proyectándose a sus fines específicos como todo ser vivo, puede desordenarse en su actividad y frustrar la consecución de sus fines u objetivos naturales; el único que, siendo parte en la sinfonía de la naturaleza, puede tocar la nota discordante. El único, pues, en cuya actividad y naturaleza hay orden y desorden. El único falible. (1972, p. 2).

Cada ser tiende al fin propio que su misma naturaleza lleva consigo. De allí que el ser humano siempre se ha preguntado por la felicidad y el sentido de su vida; el problema es que, por el solo tender hacia ese impulso perfectivo, no asegura su posesión. Su tendencia hacia la perfectibilidad lo impela, sin estar determinado hacia ella; requiere de su elección libre.

Ahora bien, es importante aclarar que en el pensamiento del autor el concepto de naturaleza humana cobra gran importancia: "Todos los seres perfectibles $-\mathrm{y}$ por ende, también el hombre- tienen como base y condicionante de su perfectibilidad a su propia naturaleza" (Ruiz Sánchez, 1962b, p. 66).

Por un lado, la falibilidad, implica imperfección, pero por el otro exige del hombre, en virtud de su propia esencia, abrirse hacia los senderos de la perfectibilidad humana. Así, la perfectibilidad constituye el segundo eje del pensamiento de Ruiz Sánchez; refiere a "aquella realidad que puede llegar a una riqueza superior en el orden de la propia entidad" (1962a, p. 178). Puede darse como una aptitud activa cuya mayor perfección le adviene o es exigida por la misma naturaleza de un ser. En consecuencia, así como la falibilidad fundamenta la necesidad de la educación en el pensamiento 
del autor, la perfectibilidad sustenta su posibilidad en tanto actúa a modo de empuje interno o dinamismo de la naturaleza del hombre hacia su plenitud, como cierta apertura hacia lo que no se posee. Sostiene el autor:

La perfección proporcionada a la naturaleza racional -y exigida por ella- no le adviene de modo fatal, por la sola puesta en marcha de su dinamismo, como le adviene en su orden al vegetal o al animal. El hombre no logra forzosamente su perfección de hombre, a la cual tiende, sin embargo, forzosamente.

Es pues la suya una naturaleza que, como tal, lleva de un modo intrínseco la tendencia a una perfección que necesita para lograrse, para ser con plenitud; pero que también de un modo intrínseco, lleva incardinada su falibilidad: en el acto de la inteligencia y en el acto libre de su voluntad. (Ruiz Sánchez, 1962a, p. 184).

Comprender el carácter de auxilio que constituye la educación desde el ángulo de la causa eficiente es concluyente para interpretar el pensamiento de Ruiz Sánchez, pues todo el fundamento antropológico es coherente con la definición de educación que propone:

El auxilio al hombre, en tanto que indigente y falible, por el cual éste puede lograr su plenitud dinámica, esto es, la capacidad estable para ordenarse libre y rectamente, en su dinamismo interior y en su autoconducción hacia los bienes individuales y comunes, naturales y sobrenaturales que plenifican su naturaleza. (Ruiz Sánchez, 1978a, p. 21).

Así se evidencia el aspecto fundamental de lo perfectivo de la finalidad educativa, dentro de la propuesta del profesor mendocino. Ahora bien, cabe preguntarse: ¿qué aspectos constituyen el proceso motivacional desde esta perspectiva?, ¿qué implica la motivación en orden al fin de la educación? Pues, como se ha manifestado, la intención es abrir el debate acerca de la posibilidad de un proceso motivacional en el orden perfectivo dentro de lo que se ha denominado la
Plenitud Dinámica, que excluye su reducción a un fenómeno de carácter pático o meramente funcional. Es en este sentido que se conceptualiza la motivación como un proceso relacional, es decir, no limitada ni reducida a uno de los aspectos del hombre -sensitivo o volitivo, como postulan muchas de las teorías actuales- ${ }^{3}$, sino como un proceso en el que se integran la propuesta de objetos buenos, la implicancia del educando desde la perspectiva metodológica y la valoración del sujeto.

\section{Aspectos del proceso motivacional perfectivo}

Si partimos de que la motivación es un proceso que relaciona todos los aspectos del educando, se requiere indagar -al menos en parte- cuáles son los mismos y qué papel juegan en el proceso educativo. Desde un criterio cronológico, se propone, en primer lugar, explicar qué significa implicar al sujeto metodológicamente desde esta perspectiva que formaliza la finalidad educativa en Francisco Ruiz Sánchez. En segundo lugar, la propuesta de objetos buenos cobra importancia en el planteo perfectivo, en tanto no es indiferente el fin educativo que se persiga. En tercer lugar, la valoración positiva del educando con respecto al objeto que lo perfecciona, sin la cual él no podrá encaminarse hacia su plenitud.

\section{La implicancia del educando desde la metodología}

Para algunos autores la motivación "no debería considerarse como una característica de una personalidad relativamente estable" sino como "un proceso psicológico en el que las características de personalidad (motivos, razones, habilidades, intereses, expectativas, perspectiva de tiempo futuro) y la percepción de las características ambientales, interactúan" (Lens, Matos y Vansteenkiste, 2008, p. 2). En cambio, desde un enfoque perfectivo se espera dos cosas. Por un lado, la tendencia hacia algo que no se posee aún. De allí que Ruiz Sánchez afirme el carácter 
dinámico de la plenitud dentro de su definición de educación, pues insatisfecho el hombre o ya perfecto de hecho, no tendría lugar esta especie de movimiento o proceso de actualización perfectivo que encierra la concepción de educación presentada. Por otro lado, es la interacción con el ambiente y con el mismo educador, la que, en lo fundamental, implica la presentación del objeto valioso en un primer momento.

Sin embargo, se advierte en la práctica docente de nuestros días una concepción motivacional restringida a un fenómeno exclusivamente metodológico-didáctico que apunta a impactar al alumno en sus facultades sensibles para lograr, luego, el aprendizaje de aquello que se presenta. Desde una propuesta que busca ser integral, como la que aquí presentamos, se reconoce que la captación sensible es solo el primer paso y que, de hecho, el proceso motivacional debe entenderse de un modo mucho más amplio, aunque comience con el sujeto implicado desde el aspecto sensorial. Si siguiéramos un criterio jerárquico en lugar de cronológico, este aspecto se ubicaría en el último lugar por ser en sí mismo el menos importante, pues qué es objeto de la motivación es superior a cómo; el segundo término se ordena al primero. No obstante, como se ha señalado, se pretende seguir el orden del proceso motivacional; en este sentido, si bien este aspecto es el menos importante, paradójicamente, es el más necesario, ya que sin el cómo (implicancia del educando desde la metodología) puede que no se llegue al qué (su actualización y valoración perfectiva).

Por ello, el fundamento antropológico adquiere gran importancia, en cuanto que va de la mano con la comprensión de cómo es y cómo se produce el conocimiento en el sujeto. En efecto, el ser humano actúa según su naturaleza ya que, como dijimos, el modo de obrar se configura en función del modo de ser. En esta dirección, se reclama que el sujeto se implique en el aprendizaje según las operaciones que le son propias en el camino del conocimiento. Como han comprobado numerosas teorías contempo- ráneas, mediante una mayor actividad del educando se logra que el aprendizaje sea mejor internalizado. Como bien expone Hernández de Lamas (2000):

Toda conducta humana parte del conocimiento y éste [sic] de la experiencia. En efecto, empezamos a conocer por los sentidos externos. Las sensaciones producidas son organizadas por los sentidos internos, que tienen una función de desmaterialización y de preparación del objeto que es presentado a la inteligencia para la abstracción.

Este principio implica que el hombre no tiene ningún tipo de conocimiento y/o estructura innata, previa a la experiencia. (p. 87).

De allí que la formación de la sensación como primer momento de conocimiento -aunque aún sensible- es primordial. Es común advertir que se recuerda aquello que mejor se aprehende con los sentidos; la experiencia constituye el primer momento de contacto entre el sujeto y el objeto de conocimiento. Por consiguiente, involucrar y hacer participar al alumno mediante los diversos métodos de aprendizaje adquiere importancia por su impacto motivacional, el cual suscita una tendencia de índole pática en el sujeto.

Como sostiene Vázquez (2012, p. 178) siguiendo a Lersch (1966) y Blanco (2002):

Podemos hablar de un 'círculo del obrar', cuyo primer paso es el conocimiento, la aprehensión-sensible, mixta o intelectual- de una realidad diversa de la del sujeto. Esas realidades son portadoras de cualidades de valor, que, si son advertidas por el sujeto, lo afectan, producen una cierta pasión, que la psicología llama respuesta afectiva, subrayando justamente su carácter pático. Esa afección debe distinguirse del subsiguiente momento activo, que es la tendencia. (Vázquez, 2012, p. 178).

Entonces, se puede sostener que el proceso motivacional perfectivo debe respetar la puerta del conocimiento, esto es, la afección 
que el nuevo conocimiento genera en nuestra sensibilidad, ese primer paso que implica una afección permitirá la posterior actualización de las operaciones llamadas espirituales -inteligencia y voluntad-.

Sin embargo, no basta involucrar al educando en este primer nivel de conocimiento sensible; es necesario, además, tener presentes las circunstancias del aprendizaje para que sean óptimas en función de promover su proceso. Aquí, una serie de elementos juegan un papel importante, como son el contexto de aprendizaje, el clima áulico, la disposición biológica y psicológica del sujeto, la apertura hacia el educador, entre otros. Por lo tanto, un método adecuado que se emplee en circunstancias desfavorables no vehiculizará el aprendizaje y disminuirá, cuando no anulará, las posibilidades de esa afección primera que se genera desde la sensibilidad mediante el método utilizado por el educador.

\section{La propuesta de objetos buenos y valiosos}

Ahora bien, en la definición de educación expresada por Ruiz Sánchez, el objeto motivacional que se presenta al alumno no es una tensión indiferente que se pueda dirigir hacia cualquier finalidad. La educación implica un perfeccionamiento del alumno en orden a su Plenitud Dinámica. En este sentido, una metodología de la enseñanza acorde a la forma connatural de conocimiento del hombre, pero "vacía" de contenido perfectivo es una visión formalista del aprendizaje, netamente academicista, que poco se vincula - o solo en parte-con una definición antropológica integral.

Por tanto, el autor considera que los objetos de conocimiento son susceptibles de ser valorados o ponderados en función de su bondad. De este modo, plantea que la perfectibilidad demanda la aprehensión o posesión de objetos buenos que plenifican al ser humano. En consecuencia, el hecho educativo cobra sentido si el educador acerca los objetos o bienes que se ordenan a dicha plenitud, mediante el método adecuado. De allí que el profesor mendocino parta de la asunción del carácter inacabado y falible con el que nace el hombre (1972, p. 1) para fundamentar la necesidad del auxilio -en términos del autor- que ofrece la educación (1978a, p. 21). El educador posee una doble tarea: por un lado, ser prudente en la presentación de bienes objetivos, reales, accesibles al educando y, por otro, forjar a su vez el hábito para que, haciendo uso de su libertad, pueda tender a ellos por propia decisión y moción.

Dado que el contacto del educando con los objetos buenos, valiosos y perfectivos debe ser real, es clara la importancia de lo que prescribe el currículo en materia educativa. Además, el diseño y contenido curricular que admita la motivación desde una visión perfectiva implica que las habilidades formales se conciban en función del objeto no solo por su uso, pues la potencia se especifica por su acto. En este sentido, la intelección se ordena a captar, apoderarse o hacer propio un nuevo conocimiento, que es el sentido etimológico del vocablo aprender (Hernández de Lamas, 2000, pp. 6-7). Así, por ejemplo, el ojo no ve solo para ver-aspecto formal-, sino en función de ver algo ${ }^{4}$.

Por consiguiente, el objeto que entra en contacto con el educando debe implicar su actualización perfectiva en los diversos órdenes, individuales y comunes, naturales y sobrenaturales (Ruiz Sánchez, 1978a, p. 21). Por lo mismo, la respuesta afectiva debe vincularse intrínsecamente con lo real, que no dejará indiferente al educando:

En la medida en que la realidad no es neutra, sino portadora de algún valor -es decir de cualidades capaces de actualizar diversas dimensiones del sujeto- es capaz de suscitar una respuesta afectiva del sujeto y así mover al sujeto a tender. La tendencia aparece fenoménicamente como un ir hacia el objeto, en busca de la unión real. (Vázquez, 2012, p. 182). 
Ahora bien, para que esa unión se produzca y el alumno se movilice hacia el objeto de conocimiento bueno y valioso para sí, debe antes presentársele de alguna manera atractiva, como ya se ha expuesto antes. Para ello, vale aclarar que el agente educativo deberá respetar las condiciones intrínsecas a la forma de conocimiento del sujeto y que, por tanto, favorecen la apropiación del objeto. En los tiempos actuales se percibe un desmesurado optimismo en la didáctica en el que se coloca "toda la esperanza", dejando de lado la discusión sobre la potencialidad perfectiva del objeto de conocimiento. Perkins (2001) critica el "síndrome del salvador", el que revela cierta impaciencia por alcanzar la solución rápida que pondrá todo en orden en la escuela. Así, para el autor, la decisión más importante radica en el currículum, en elegir qué hemos de enseñar (Perkins, 2001, p. 53). Recién en un segundo momento cabe considerar la cuestión del método.

El hábito entra de lleno en la perspectiva abordada desde sus dos aristas, ya sea como hábito formal o como hábito material. Esto es, en el primer caso, el ejercicio de la potencia sin referencia al objeto y, en el segundo, la actualización de la potencia en relación con un determinado objeto, aunque "en el hábito están siempre presentes las dos dimensiones, inescindibles, bien que a veces se acentúe uno u otro aspecto" (Vázquez, 2012, p. 165). De aquí es que podemos unir el aspecto metodológico con el hábito formal y el motivo u objeto de tal motivación con el hábito material. Tales aspectos componen la integridad y unidad de la dinámica sujeto-proceso motivacional perfectivo. Por consiguiente, prescindir del contenido valioso implicaría desvincular al educando del carácter teleológico de la educación.

Mediante la actualización perfectiva del sujeto a través del objeto se va forjando el hábito, el que "establece un orden interior que favorece al acto libre, no porque lo produzca, sino porque lo permite, como condición necesaria, aunque no suficiente" (Vázquez, 2012, p. 164).
Dicho orden interior es una unificación del ser, es decir, de todas sus potencias en función de esta teleología perfectiva que es el motivo último de la tarea educativa (ya sea como auto o hetero-educación).

\section{La concienciación del valor del objeto}

Ahora bien, el cómo -método-, es importante para llegar al qué, pues el solo hecho de presentar un objeto valioso y bueno en orden a la plenitud del sujeto no alcanza, por lo que es preciso entender la vinculación señalada entre la potencia y el acto.

La realidad en cuanto es valorada por el educando, no es percibida ya al modo que se da en sí misma, sino que es asimilada o conocida según los propios esquemas valorativos personales y los influyentes del entorno; de ello resulta una relación compleja y única, propia de cada sujeto.

La propuesta de un objeto perfectivo puede no ser determinante de su elección. En efecto, el alumno puede tener por delante un objeto perfectivo y no ser consciente de su valía o, también, puede ser consciente de su valor y aun así no elegirlo, dado que el hombre no tiende forzosamente a sus bienes, sino de manera libre. De aquí la necesidad de hacer consciente lo que implica la incorporación perfectiva: "toda motivación está precedida de una adecuada preparación cognoscitiva [y] si se pretende ampliar el horizonte -tanto temporal como cualitativo- del tender, es preciso presentar para su conocimiento las dimensiones valiosas de lo real" (Vázquez, 2012, p. 178).

Esta manera relacional de presentar la motivación como un proceso psicológico que engloba la unidad del educando con diversos objetos perfectivos en orden a un fin plenificador -Plenitud Dinámica- nos devuelve una mirada integral sobre el hombre. En efecto, la tendencia no se presenta solo como un aspecto neutro o meramente formal, sino dotado de 
sentido en función de un fin que confiere significado a todo el proceso.

La concepción de la motivación que hemos planteado a partir de la teoría pedagógica del profesor mendocino supera algunas de las propuestas motivacionales que parecen vincularse con teorías homeostáticas y con teorías que la conciben como un mero estado de activación mediante un estímulo externo, cuestión que en parte se halla subsumida en el aspecto metodológico que aquí presentamos, en consonancia con los planteamientos de Vázquez (2012).

En suma, el eje motivacional que aporta el análisis consiste en la adhesión a un fin perfectivo consecuente con la naturaleza humana respecto del cual no basta la sola ejecución de las potencias (ya sean sensibles o espirituales), sino que exige la unidad de estas a objetos cuya incorporación actualice constantemente al sujeto y lo perfeccione. La concienciación valorativa es fundamental, ya que permite al educando captar lo valioso y reconocer, en consecuencia, el carácter del objeto al cual tiende.

De este modo, la motivación no es solo una razón, idea o motivo del obrar humano; es un proceso de índole psicológico-relacional que cobra importancia ante la advertencia y adhesión al valor de los objetos que plenifican la existencia en sus diversos órdenes.

\section{Reflexiones finales}

Hemos mostrado, al seguir los estudios de Ruiz Sánchez, que el fin en su carácter teleológico actúa como eje que posibilita la selección y unificación de los objetos que perfeccionan las potencialidades del alumno.

Gracias al fin, los objetos son valorados en su carácter moral y seleccionados en pos de su incorporación perfectiva. La Plenitud Dinámica, en cuanto razón última de toda tarea educativa, da respuesta a la falibilidad del hombre y lo encamina hacia su perfección mediante el auxilio pedagógico.

En la enseñanza, la motivación transciende los límites del proceso homeostático o la sola función ejecutora de las potencias para trasladarse al terreno valorativo que impele a la propia existencia en su devenir perfectivo. La comprensión del proceso motivacional incorpora la falibilidad, perfectibilidad, libertad y Plenitud Dinámica. Por eso es una propuesta que supera algunos reduccionismos de las teorías motivacionales contemporáneas y que abre el debate acerca de la posibilidad de considerarla como una visión de este importante elemento del proceso educativo que integra armónicamente todas las dimensiones del ser humano.

Por último, nótese que para esta propuesta corresponde al educador mostrar al alumno esa realidad pasible de afectarlo, de modo que se produzca en él la respuesta afectivo-tendencial deseada. La motivación educativa, en función de los estudios mencionados, ubica al docente en un lugar central del proceso de enseñanza y aprendizaje, pero no omnipotente: es para Ruiz Sánchez el facilitador del proceso perfectivo. Desde el análisis efectuado se podrían proponer como nueva línea de investigación un abordaje comparativo entre la perspectiva motivacional realista -como la que hemos presentado desde Ruiz Sánchez- y otros posicionamientos actuales, como las teorías que tienen que ver con el compromiso académico (engagement) (Sandoval-Muñoz et al., 2018; Daura, 2015; Cole y Korkmaz, 2010). Asimismo, sería oportuno elegir una de las teorías motivacionales - como la de motivación de logro, de la atribución, del control de la acción o de la autodeterminación- y analizar, por un lado, el fin educativo que propone o se puede inferir de ella $y$, por otro, el valor central en que se formaliza, ya que aquí solo nos hemos limitado a señalar la ausencia del carácter perfectivo en general. En un estudio de mayor amplitud, se podrían analizar los enfoques mencionados desde los supuestos epistemológicos que sostienen muchas 
de las teorías contemporáneas de la Psicología de la Educación (Portela, Boarini, Di Marco y González, 2018).

En el plano aplicado, resultaría de interés implementar un estudio de campo en centros educativos que trabajen los elementos que componen la motivación perfectiva y analizar así el impacto de sus resultados.

\section{Referencias}

Atkinson, J. W. (1964). An Introduction to motivation. Princeton, United States: Van Nostrand.

Atkinson, J. W. (1974). Motivation and achievement. New York, United States: Holt.

Barrio Maestre, J. M. (2004). Elementos de antropología pedagógica (3. ${ }^{\mathrm{a}}$ Ed.). Madrid, España: Rialp.

Blanco, G. (2002). Curso de Antropología Filosófica. Buenos Aires, Argentina: EDUCA.

Cecchini, J. A., Méndez-Giménez, A., y García Romero, C. (2019). Relationships between $3 \times 2$ achievement goals and the satisfaction of the basic psychological need for competence. Revista de Psicodidáctica, 24(1), 53-61.

Cole, J. S., y Korkmaz, A. (2010). Using Longitudinal Data to Improve the Experiences and Engagement of First-year Students. New Directions for Institutional Research, 2(Esp.), 43-51. doi: 10.1002/ ir.371

Daura, F. (2015). El estudio del compromiso académico. Panorama general sobre su abordaje. Diálogos Pedagógicos, 13(25), 54-75.

Deci, E., y Ryan, R. (1985). Intrinsic motivation and self-determination in human behavior. New York, United States: Plenum.
Deci, E., y Ryan, R. (2000). The 'What' and 'Why' of Goal Pursuits: Human Needs and the Self-Determination of Behavior. Psychological Inquiry, 11(4), 227-268.

Di Marco, M. E., Portela de Nieto, A. I., González, M. L., Boarini, M. G., y Difabio de Anglat, H. E. (2018). El hecho educativo desde la perspectiva de Francisco Ruiz Sánchez. Contribuciones para su comprensión pedagógica-didáctica. Dilemas contemporáneos: Educación, Política y Valores, VI(1), 1-21.

Elliot, A., y McGregor, H. (2001). A 2x2 Achievement goal framework. Journal of Personality and Social Psychology, 80, 501-519.

Gadamer, H. G (1994). Verdad y método. Salamanca, España: Sígueme.

Gadamer, H. G. (2015). Verdad y Método II (9. ${ }^{\mathrm{a}}$ Ed.). Salamanca, España: Sígueme.

García Hoz, V. (1962). Cuestiones de filosofía individual y social de la educación. Madrid: Rialp.

Heckhausen, H. (1991). Motivation and action. Berlin: Springer-Verlag

Hernández de Lamas, G. B. (2000). Los desafios del aprendizaje. Buenos Aires: Instituto Mater Dei.

Kuhl, J. (2000). A functional design approach to motivation and self-regulation: The dynamics of personality systems and interactions. En M. Boekaerts, P. Pintrich у M. Zeidner (Eds.), Handbook of selfregulation (pp. 111-163). San Diego, CA, United States: Academic.

Kuhl, J., y Beckman, J. (1985). Action control: From cognition to behavior. New York, United States: Springer-Verlag.

Lens, W., Matos, L., y Vansteenkiste, M. (2008). El profesor como fuente de motivación de los alumnos: Hablando del 
qué y del por qué del aprendizaje de los alumnos. Revista Digital de Investigación en Docencia Universitaria, 4(1), 1-9. doi: 10.19083/ridu.4.9

Lersch, P. (1966). La estructura de la personalidad. Barcelona, Espaa: Scientia.

Maritain, J. (2008). La educación en la encrucijada. (Trads. C. Segade y J. M. Garrido). Madrid, España: Palabra, S.A.

Millán Puelles, A. M. (1983). La formación de la personalidad humana. Madrid: Rialp.

Niemiec, C., Ryan, R., y Deci, E. (2010). Self-Determination Theory and the Relation of Autonomy to Self-Regulatory Processes. En R. Holy (Ed.), Handbook of Personality and Self-Regulation (pp. 169-191). Singapore: Wiley-Blackwell.

Nuttin, J. (1973). La motivación. En J. Nuttin, P. Fraisse y R. Meili, Motivación, emoción $y$ personalidad. Buenos Aires, Argentina: Paidós.

Nuttin, J. (1980). Motivation et perspective d'avenir. Lovaina, Bélgica: Presses Universitaires.

Perkins, D. (2001). La escuela inteligente. Del adiestramiento de la memoria a la educación de la mente (2. reimp.). Barcelona: Gedisa.

Ruiz Sánchez, F. (1962a). Perfectibilidad y Educabilidad. Sapientia, XVII(65), 178-192.

Ruiz Sánchez, F. (1962b). El concepto del hombre y la educación. Boletín de Estudios Políticos y Sociales, 12, 65-80.

Ruiz Sánchez, F. (1972). Acerca de la Educabilidad. Cuadernos de Pedagogía, 1, Mendoza, Facultad de Filosofía y Letras, Universidad Nacional De Cuyo.

Ruiz Sánchez, F. (1973a). Esquema tentativo para una estructuración de la temática fundamental de la Pedagogía. Mendoza:
Instituto de Filosofía, Facultad de Filosofía y Letras, Universidad Nacional De Cuyo.

Ruiz Sánchez, F. (1973b). Los Fines de la Educación. Cuadernos de Pedagogía, 2. Mendoza, Facultad de Filosofía y Letras, Universidad Nacional de Cuyo [Parcialmente incorporado en la segunda parte de su obra Fundamentos y Fines de la Educación].

Ruiz Sánchez, F. (1978a). Fundamentos y Fines de la Educación. Mendoza: Facultad de Filosofía y Letras, Universidad Nacional de Cuyo.

Ruiz Sánchez, F. (1978b). Introducción a los fundamentos antropológicos de la educación. Actas de las Primeras Jornadas $\mathrm{Na}$ cionales Universitarias de Filosofía de la Educación (pp. 39-58). Paraná, Universidad Nacional de Entre Ríos.

Sandoval-Muñoz, M. J., Mayorga-Muñoz, C. J., Elgueta Sepúlveda, H. E., Soto Higuera, A. I., Viveros Lopomo, J., y Riquelme Sandoval, S. V. (2018). Compromiso y motivación escolar: Una discusión conceptual. Revista Educación, 42(2), 1-14.

Portela, A. I., Boarini, M. G., Di Marco, M. E., y González, M. L. (2018). El problema de la brecha epistemológica en las teorías psicoeducativas. El Guiniguada, $27,71-82$.

Vázquez, S. M. (2009). Motivación y voluntad. Revista de Psicología, 27(2), 185-212.

Vázquez, S. M. (2012). La Filosofía de la Educación. Estado de la cuestión y lineas esenciales (2. ${ }^{\mathrm{a}}$ Ed.). Buenos Aires, Argentina: CIAFIC.

Villalba, M. (2001). Inferencia y conocimiento previo de lógica. Relaciones y dependencias. Sapientia, LVI, 251-264. 
Weiner, B. (2010). The Development of an Attribution-Based Theory of Motivation: A History of Ideas. Educational Psychologist, 45(1), 28-36.

\section{Notas}

${ }^{1}$ El autor sostiene que los fines del hombre objetivamente considerados son: Dios, el bien común político, el bien común familiar $\mathrm{y}$ el bien individual.

2 Otros autores, como Millán Puelles (1983), García Hoz (1962), Maritain (2008), Barrio Maestre (2004), entre tantos más que comparten la misma tradición de pensamiento, se refieren al mismo hecho en otros términos: limitación, fragilidad de la naturaleza humana, inacabamiento, finitud, falencia, imperfección, etc.
${ }^{3}$ Para ampliar sobre las teorías contemporáneas de la motivación y sus posibles reduccionismos remitimos a Vázquez (2009).

${ }^{4}$ En este sentido es muy valioso el análisis de M. Villalba (2001) sobre la pérdida de la función intelectiva de la razón humana en relación con la preponderancia de teorías pedagógicas que postulan una función meramente instrumental para la misma. 
